\title{
FEMINISTAS EN EL FORO
}

\author{
LILIAN CELIBERTI \\ Articulación Feminista Marcosur \\ VIRGINIA VARGAS \\ Articulación Feminista Marcosur
}

\begin{abstract}
Resumen: El articulo trata sobre la forma de incursión de expresiones significativas de la pluralidad feminista en el Foro Social Mundial expresa. Estas incursiones expresan los cambios en las subjetividades y en las estrategias de lucha que comienzan a desarrollar los movimientos feministas en particular y los movimientos sociales en general, en el marco de un mundo globalizado y en el nuevo milenio. Es un proceso inédito, que esta impulsando el desarrollo de nuevos paradigmas para la acción colectiva, que combina lo local y lo global, la interconexión de múltiples agendas y la recuperación de una dimensión mas profunda de la justicia económica, social, cultural y simbólica, ampliando, en este proceso, el concepto de la política, lo político y el poder. El articulo coloca en debate las formas de hacer política de los movimientos sociales que confluyen en el Foro - que arrastran viejas dinámicas y al mismo tiempo recrean los nuevos paradigmas - y que abren la posibilidad de reinventar un mapa emancipatorio $y$ un imaginario social, capaz de competir con el consenso neoliberal y el pensamiento único, recuperando la diversidad y la pluralidad de sujet@s y actor@s sociales.
\end{abstract}

Palabras claves: globalización alternativa, cultura politica, utopia, nuevos paradigmas, agendas feministas.

El Foro Social Mundial (FSM) expresa las esperanzas, las búsquedas y propuestas de los movimientos sociales que cuestionan la globalización neoliberal y los dramáticos procesos de exclusión. Se ha convertido en un espacio de confluencia de las luchas y propuestas de movimientos, organizaciones, redes, campañas, múltiples actores y actoras que han asumido ese espacio como propio, avanzando nuevas perspectivas para un pensamiento utópico, algo casi perdido en el horizonte social de las ultimas décadas. Y esta es su riqueza y su fortaleza. Concebido no como evento sino como proceso colectivo de redes, coaliciones, campañas, alianzas y movimientos, el FSM alimenta presencias y disputas en el espacio global.

La experiencia desde el primer Foro Social mostró sus potencialidades para colocar en debate los principales desafíos del mundo e impulsó un proceso que contribuyó a

Copyright (c) 2003 by Revista Estudos Feministas 
enriquecer las articulaciones y habilitar la formulación de propuestas. Como espacio en construcción, el FSM está sujeto "a interpretaciones y presiones acerca de su sentido y su futuro. Es un espacio de debate de ideas, propositivo y al mismo tiempo de movilización, acción y lucha. Es un espacio contestatario, que manifiesta inconformismo. Allí se juntan los organizados, pero también es un espacio aglutinador de quienes quieren ser parte de este proceso, sin identidad de organización o filiación ideológica o partidaria. El FSM es un espacio democrático, aglutinador de fuerzas progresistas colectivas e individuales", como dice Sergio Hadad. ${ }^{1}$

Esta diversidad demostró, desde el primer momento, que sus alcances no son fáciles de sintetizar y, aún más importante, que cualquier pretensión de hacerlo conlleva el riesgo de empobrecimiento y frena su propio desarrollo. Para avanzar en la idea de proceso de confluencia, el Comité Organizador (después del primer Foro), impulsó la creación de un Comité Internacional con el objetivo de "mundializar el Foro" y puso en discusión las bases para una "Carta de principios" que estableciera pautas y fronteras del espacio a construir.

La Carta de Principios" definida en el Comité Internacional del FSM en junio del 2001 , establece el marco ético-político de pluralidad, diversidad y reconocimiento, como ejes para multiplicar y ampliar el espacio del FSM.

Al considerar que el FSM no es sólo un evento sino principalmente un proceso cuyo logro fundamental es, justamente, ser percibido como proceso colectivo de redes, coaliciones, campañas, alianzas y movimientos, coloca en el campo social la esperanza de construir nuevas culturas políticas. Sin carácter decisorio, sin mayorías o minorías, se comienza a consolidar un espacio diverso, plural, no gubernamental, no confesional, descentralizado, movimientista y sin ninguna pretensión de representar todas las iniciativas impulsadas por una sociedad civil global en formación. "Nadie estará autorizado a expresar, en cualquiera de sus encuentros, posiciones que pretendan ser representativas de todos sus participantes (...) El Foro por lo tanto, no se constituye en una instancia de poder, a ser disputado por los participantes de sus reuniones, ni pretende constituirse en única alternativa de articulación o acción de las entidades y movimientos que en él participan". La Carta de Principios establece también una frontera para la participación: "No deben participar del Foro representaciones partidarias, ni organizaciones militares". ${ }^{3}$

Estos principios constituyen un marco fundamental para el desarrollo del debate político. Sin embargo, en la misma medida en que el Foro crece como espacio simbólico, la tentación de "capitalizar" - en el sentido más tradicional del término - un tan vasto movimiento se expresa principalmente en debates organizativos que manifiestan la tensión cada vez más evidente entre "representación" y "participación". ¿Qué grado de centralidad y conducción puede adquirir este nuevo movimiento de búsqueda de alternativas al neoliberalismo? ¿Cómo se expresa la diversidad y pluralidad? Hay quienes imaginan el FSM como un movimiento de movimientos y quienes lo piensan como un espacio de convergencia que habilita el desarrollo de múltiples espacios de acción y formulación de propuestas y, por tanto, más un "campo" de actor@s que un lugar de pronunciamiento y definición de estrategias.

El Comité Internacional es uno de los escenarios de la disputa de "interpretaciones y presiones" acerca del sentido y el futuro del FSM, como señalaba Sergio Haddad. Para algunos actores, el Foro Social Mundial es un espacio de confluencia de la lucha

\footnotetext{
1 Sergio Hadad, integrante del Comité Organizador del FSM, en: Evaluaciones del FSM 2002 www.forumsocialmundial.org.br.

${ }^{2}$ Ver Carta de Principios en: www.forumsocialmundial.org.br.

${ }^{3}$ Carta de Principios Foro Social Mundial - www.forumsocialmundial.org.br.
} 
antiglobalización donde concertar una agenda de movilizaciones globales; para otros es un espacio plural donde es posible compartir y articular alternativas democráticas y democratizadoras.

Para nosotras, como Articulación Feminista Marcosur, el FSM es un espacio que tiene como principal desafío el desarrollo de nuevas culturas políicas, garantizando la expresión de un amplio campo de actores y actoras desde la diversidad y pluralidad, que abre la posibilidad de colocar en diálogo diferentes movimientos, identidades y agendas.

Como expresa Judith Butler: "A diferencia de una visión que forja la operación de poder en el campo político exclusivamente en términos de bloques separados que compiten entre sí por el control de las cuestiones políticas, la hegemonía pone el énfasis en las maneras en que opera el poder para formar nuestra comprensión cotidiana de las relaciones sociales y para orquestar las maneras en que consentimos (y reproducimos) esas relaciones tácitas y disimuladas del poder (...) Más aún, la transformación social no ocurre simplemente por una concentración masiva a favor de una causa, sino precisamente a través de las formas en que las relaciones sociales cotidianas son rearticuladas y nuevos horizontes conceptuales abiertos por prácticas anómalas y subversivas". ${ }^{4}$

En tal sentido, el FSM es un espacio de práctica del diálogo y encuentro entre diferentes visiones, estrategias y agendas. Romper la dicotomía entre economía y sociedad, economía, naturaleza y sociedad, reinventar un mapa emancipatorio que se construya desde las luchas e identidades particulares y no en contra de ellas, reinventar un imaginario social pleno capaz de competir con el consenso neoliberal y el pensamiento único, convocando a la diversidad y la pluralidad de sujet@s y actor@s, parece el camino más difícil y sin duda más desafiante.

\title{
Impacto de la globalización en los "sujetos feminisłas"
}

\begin{abstract}
"Son los marcos los que dan significado a eventos y acontecimientos, le dan sentido al mundo, organizan la experiencia y guían la acción individual y colectiva. Los marcos son las metáforas, representaciones simbólicas y claves cognitivas que modelan los comportamientos y ayudan a evaluar los acontecimientos. Es claro que los marcos no son permanentes o estables. Tampoco son consensuados o únicos. En todo momento histórico, distintos marcos interpretativos pueden competir entre sí, acompañando o aun promoviendo conflictos entre actores, desafiando interpretaciones hegemónicas y sugiriendo cursos de acción alternativos."
\end{abstract}

En este nuevo contexto, los movimientos se enfrentan a tendencias ambivalentes. La globalización impulsa tendencias homogeneizadoras, reestructurando las sociedades y las formas en las que los individuos se relacionan con los cambios estructurales y subjetivos. Se producen fragmentaciones y rearticulaciones en una nueva forma organizativa, lo que Castells ha llamado la "sociedad de redes", ${ }^{6}$ con conexiones amplias y cosmopolitas. Este dominio de redes tiene una "... multiplicidad abrumadora de combinaciones de intercambios de bienes tangibles e intangibles... que fluyen a través de una mirada de nodos y canales identificables que interconectan a los grupos sociales a través de todo el mundo. Las interacciones en el dominio de las redes comprenden a toda clase de organizaciones... cuyas interrelaciones crean enmarañadas redes de redes entrecruzadas

\footnotetext{
${ }^{4}$ Judith BUTLER, Ernesto LACLAU y Slavoj ZIZEK, 2003, p. 20.

${ }^{5}$ Elizabeth JELIN, 2003, p. 40-41.

${ }^{6}$ Manuel CASTELLS, 1999.
} 
entre sí, superponiéndose unas a otras y en constante transformación". Se generan así nuevas conexiones, nuevos canales y nodos, destruyendo los viejos y sufriendo en este proceso un sin fin de mutaciones y evoluciones. ${ }^{7}$

En este concierto de conexiones y entrecruzamientos, los movimientos sociales ven modificada su existencia y se expresan en forma diferente. No como actores unificados ni solamente como movimientos de contenido plural. Se expresan más bien como un "campo de actores", amplio, diverso y en permanente ampliación y transformación. ${ }^{8}$ Otras autoras, como Sonia Alvarez, reconceptualizan los movimientos sociales (en referencia a los feminismos) como un campo discursivo, expansivo, heterogéneo, generando campos de acción policéntricos que se extienden sobre un conjunto distintivo de organizaciones de la sociedad civil, ${ }^{9}$ construyendo públicos alternativos que resignifican y confrontan los sentidos políticos culturales dominantes en la sociedad.

En este campo heterogéneo, las formas de resistencia varían. Se multiplican los sitios de intervención conectando y coordinando la voluntad colectiva de miles a través de intercambios electrónicos. Las identidades se vuelven "nómades" 10 porque se movilizan permanentemente, evitando la fijación en una sola. Salvo en los fundamentalismos, donde el miedo a la exclusión se expresa en una búsqueda permanente de certezas, a través del posicionamiento de una identidad como verdadera y excluyente.

Los feminismos llegan a este proceso global también en forma diferente a la del pasado. Es decir, no desde una identidad única, no desde una hipotética "hermandad feminista global", que descontextualiza y despolitiza su presente, al aludir a una forma compartida y hegemónica de ser mujer, ni a una única forma de posicionarse como feministas. Los marcos simbólicos y discursivos son mucho más amplios y movibles. En esta realidad, donde la diversidad y heterogeneidad es la que prima, quizás no sea tan importante un programa definido y consensuado de transformaciones políticas, sociales y económicas, sino la explicitación y combinación de las agendas desde colectivos diversos y de múltiples autonomías. ¿Cuál seria entonces la forma de unidad colectiva que permite respetar y desplegar las diferencias? Las formas de organización demasiado rígidas y pesadas no responden a la exigencia de una individualidad de perfiles abiertos. Tal vez estén emergiendo nuevas formas de lo colectivo, más flexibles, livianas y fugaces.

\section{Las nuevas dinámicas de intervención feministas}

Muchas de las múltiples dinámicas feministas en América Latina comienzan a orientarse hacia la nueva realidad. El Encuentro Feminista Latinoamericano y del Caribe, realizado en Costa Rica en diciembre del 2002 (el noveno desde 1981), tuvo como eje la globalización. Ello es más que significativo, en la medida en que en este espacio de los encuentros los feminismos regionales toman el pulso a sus diversidades, avances, expresan sus hallazgos, búsquedas, nudos y nuevas subjetividades, en suma, sus nuevos y múltiples derroteros en reflexión y acción. A su vez, redes globales como DAWN y AWID, Mujeres Viviendo Bajo Leyes musulmanas, entre muchas otras, vienen incidiendo desde hace un tiempo en esta nueva realidad, en articulación también con esta dimensión global que es la región latino-caribeña.

De todas estas nuevas dinámicas y espacios, nos interesa analizar la incidencia feminista en el Foro Social Mundial, espacio donde confluyen muchas de las nuevas

\footnotetext{
${ }^{7}$ Francisco SAGASTI, 1998.

8 JELIN, 2003.

${ }^{9}$ Sonia ALVAREZ, 1998, p. 295.

${ }^{10}$ Rosi BRAIDOTI, 2000.
} 
estrategias y preocupaciones de los movimientos sociales globalizados, como el feminismo. Es también un complicado sitio de alianzas con otros movimientos, cuya orientación hacia los feminismos no siempre es de reconocimiento.

\title{
Los feminismos en el Foro Social Mundial
}

\begin{abstract}
"Otro mundo es posible", es el lema del Foro Social Mundial. Mirando de una perspectiva femenina la tarea es bastante más gigantesca de lo que parece. Sin duda, estamos incomodando al pensamiento único dominante. ¿Pero estamos incomodándonos, nosotros mismos, con nuestro machismo, racismo y otras intolerancias? La especificidad del Foro Social Mundial es establecer el diálogo entre los diversos. Esto da originalidad y fuerza al Foro en la construcción de una globalización de las ciudadanías en el Planeta Tierra. Pero el camino es largo y lleno de obstáculos. Espero que las mujeres nos hagan ser radicales, actuando como hasta ahora: haciendo cobranzas e incomodando."
\end{abstract}

La presencia de los feminismos en el FSM es un aporte y un desafío. Los cambios en las subjetividades han impactado también a los feminismos y sus agendas de transformación, reincorporando a ellos las "agendas olvidadas" o debilitadas en la larga marcha hacia el fortalecimiento institucional. Una agenda que busca integrar la justicia de género con la justicia económica, recuperando al mismo tiempo la subversión cultural y la subjetividad como estrategia de transformación de más largo aliento. A esta lucha por la justicia los feminismos comienzan a incorporar la diversidad no sólo en la vida de las mujeres sino en su estrecha relación con las características multiculturales y pluriétnicas de nuestras sociedades, sin abandonar las luchas históricas por la autonomía, física, socioeconómica, política y cultural. Estas luchas expresan dos tipos de injusticia: la injusticia socioeconómica, arraigada en las estructuras políticas y económicas de la sociedad, y la injusticia cultural o simbólica, arraigada en los patrones sociales de representación, interpretación y comunicación. Ambas injusticias cruzan a las mujeres y otras identidades, raciales, étnicas, sexuales, geográficas. Expresadas en una desigual distribución de recursos y aún escasa valoración, se concretan cada vez más, en luchas por redistribución y por reconocimiento, "... porque las normas androcéntricas y sexistas se institucionalizan en el Estado y la economía, y las desventajas económicas de las mujeres restringen su 'voz' impidiendo su igual participación en la creación cultural". ${ }^{12}$

Los feminismos llevan también al FSM lo que ha sido una práctica intrínseca a su existencia y su desarrollo: sus articulaciones internacionalistas, acumuladas a lo largo de 30 años de existencia en su segunda oleada. Como dice Waterman, no hay duda del aporte de las pensadoras feministas de los 70 y los 80 al movimiento de justicia global, tanto por su experiencia internacionalista como porque "... mucho de esa reflexión sobre el nuevo momento... y comportamiento... puede ser rastreado desde las prácticas feministas internacionales previas". ${ }^{13}$

Pero las condiciones para ese internacionalismo han variado dramáticamente. Las luchas por el reconocimiento fueron el eje articulador de este internacionalismo en las décadas pasadas. No podía ser de otra manera porque los feminismos debían salir de la invisibilidad y expresar su presencia y propuesta. Esa dimensión no se puede perder. Sin embargo, los marcos interpretativos para la acción son otros y las oportunidades y riesgos también. Los actores preexistentes y los nuevos que van surgiendo tienen como reto

\footnotetext{
1 Cándido GRZYBOWSKY, 2002.

${ }^{12}$ Nancy FRASER, 1997, p. 33.

${ }^{13}$ Peter , 2002, p. 5.
} 
posicionarse propositiva y articuladamente frente a esta nueva realidad, que no deja escoger: para consolidar el reconocimiento se requiere de la redistribución y para que esta sea justa e inclusiva se requiere reconocimiento. Como dice Jelin, quien no considere esta nueva realidad "quedará rezagado y perderá el tren de la historia". ${ }^{14}$

En este intento de no quedar fuera de la historia, han surgido un sinnúmero de articulaciones e iniciativas alrededor de los nuevos retos que trae la justicia económica y las políticas de reconocimiento, dando cuenta también de las nuevas dinámicas y formas de existencia de los feminismos. Por una parte, se abren nuevos ejes de reflexión acerca de las dinámicas macroeconómicas y macropolíticas, el proceso mismo de globalización, lo global y lo local, a la vez que se crean nuevas formas de articulación, más flexibles y horizontales, con mayor capacidad de reflexión y de propuesta y que comienzan a producir análisis mas finos sobre los impactos de género de estos procesos y mega tendencias que trae la globalización (Iniciativa Feminista Cartagena y Articulación Feminista MARCOSUR, por nombrar dos de las mas recientes, que a su vez agrupan un conjunto de activas redes y organizaciones).. Hay también otras formas de articulación feminista globales como la Marcha Mundial de Mujeres, además de los aportes de algunas redes históricas, como $D A W N$, a esta nueva realidad. En todas estas articulaciones hay un conjunto significativo de feministas jóvenes, diversificando liderazgos individuales y colectivos. Estas nuevas articulaciones, presentes en el FSM, lograron colocar las perspectivas feministas en las agendas del Foro, y garantizar la presencia feminista en algunos de los debates más importantes.

Sin embargo, junto con este aporte sustancial, viene el desafío que para los feminismos representa este espacio que, al mismo tiempo que flexibiliza, arrastra viejas exclusiones. Construido desde nuevas miradas y sensibilidades, el FSM expresa también los ejes de discriminación y exclusión con relación a las mujeres. El FSM representa una articulación dialéctica entre el movimiento de justicia global y el movimiento feminista en particular. ${ }^{15}$ Esta articulación no es fácil, como dice Sonia Correa, porque implica una doble estrategia: comprometerse con las luchas colectivas de los movimientos sociales y, al mismo tiempo, intentar transformar su perspectiva en relación al feminismo, a la diferencia, al género, a los pensamientos múltiples. ${ }^{16}$ Existen por tanto en el Foro procesos de articulación y procesos de disputa. Y ello ha sido claro a lo largo de los tres Foros: - pasando de una presencia numérica significativa (52\%) pero invisibilizada en el I Foro, a una presencia mucho mas visible e impactante en el II (menos en los paneles, que muy pocos estuvieron organizados por las redes feministas, y más por el lúcido impacto que significo la "Campaña contra los fundamentalismos); logrando en el III Foro visibilidad y responsabilidad en la organización de los paneles de dos de los cinco ejes temáticos: (el de Derechos Humanos, a cargo de la Marcha Mundial de Mujeres y el de "Democracia, sociedad civil y poder político", a cargo de la Articulación Feminista Marcosur. ${ }^{17}$ Todo ello gracias a la tenacidad de la disputa planeada por los feminismos, así como a la labor incansable de los feminismos brasileños.

La integración de la Articulación Feminista Marcosur al Comité Internacional del FSM nos introduce también en una dinámica más concreta de debate, tanto en lo que se refiere al debate político específico como a la dedicación de esfuerzos en la línea de

\footnotetext{
14 JELIN, 2001.

${ }^{15}$ WATERMAN, 2002.

16 Sonia CORREA, 2002.

${ }^{17}$ Cada uno de estos ejes implicaba la organización de seis o siete paneles, cada uno con la participacion de al menos seis panelistas, de diferentes regiones, sexos, étnias, razas, orientaciones sexuales, edades.
} 
construcción del espacio del FSM, asumiendo los desafíos que ello implica.

Crear las condiciones para un debate político es tal vez uno de los principales obstáculos, éste no se da espontáneamente y muchas veces se falsea por vía de discusiones organizativas que denota una puja de poder y reconocimiento, expresa la fragmentación y la desconfianza que aún subsiste entre los diversos actores de este proceso.

Pero también nos plantea el desafío de articular diversas escalas de actuación entre lo local y lo nacional, donde se insertan las integrantes de la articulación, y la global, donde se disputan sentidos de la acción política. ¿Cómo se articulan realmente estas escalas? ¿Cómo se procesan los aprendizajes políticos de esta experiencia y cómo se expresan en lo local/nacional?.

EI FSM abre nuevos y viejos debates en el movimiento feminista. Por un lado, es un movimiento plural que se expresa en múltiples redes y espacios diferentes, con dificultades para reconocerse a sí mismo y abordar sus diferencias como parte del debate político y la búsqueda de alternativas. Y, a la vez, tiene la necesidad de aparecer y visibilizarse para que sus temas no desaparezcan. En algunos de los talleres realizados en el FSM donde se abordaron los nuevos retos feministas, se señalaron las dificultades y problemas que aún persisten en los espacios de articulación entre diferentes movimientos. "Las feministas siempre han tratado de influir en la agenda de los movimientos sociales y políticos progresistas para cambiar la perspectiva de estos movimientos. Sin embargo sabemos que queda un largo camino por recorrer para que la inclusión de la perspectiva feminista sea una realidad. [...] No queremos que el FSM se convierta en un asunto dominado por los hombres: se necesita liderazgo feminista y la construcción de alianzas [...] Este desafío es complejo. No queremos una presencia nominal y estamos cansadas de referencias superficiales a los temas de género", expresa Irma van Dueren. Ana Irma Rivera, en el mismo taller, señala que "las mujeres en términos generales, no somos voces presentes en los temas económicos ni de finanzas. Entiendo que estos temas se identifican como masculinos, no sólo en término de las voces sino también de los contenidos. Es decir que el problema de las ausencias femeninas no se resolverá sólo con la llegada de más mujeres a la discusión de los temas económicos y de finanzas sino que también es necesario que estos temas sean vistos con perspectiva de género". ${ }^{18}$

Para Sylvia Borren el tema central se ubica en la necesidad de pensar alternativas globales y potenciar liderazgos feministas: "Creo que ha llegado el momento de que muchas de nosotras (más de las que actualmente lo hacemos) tomemos una seria responsabilidad en dar forma a nuestro mundo en los niveles micro, medio y macro. En otras palabras, de dar un paso más en el feminismo, el movimiento feminista y el liderazgo feminista; de preocuparnos no sólo por la forma de vida de las mujeres sino también de la calidad de las vidas de los hombres, mujeres y niños/as en un sentido más general; de preocuparnos por encontrar soluciones a las tensiones y conflictos en el mundo; de asumir el liderazgo en organizaciones, de buscar formas de hacer que nuestras vidas y nuestro mundo sean más inclusivas y diversas".

\footnotetext{
${ }^{18}$ Las opiniones de diferentes feministas expresadas en un taller del FSM 2002 fueron publicadas por ALAl en Irene , 2002.
} 


\section{Un espacio global de los movimientos sociales: desafíos y tensiones}

Para cada persona que participa en el Foro, la creatividad, el respeto y la diversidad constituyen el patrim onio más destacado de la experiencia subjetiva vivida durante esos cinco días, en esa espacie de torre de Babel de lenguas, colores, imágenes, gestos y símbolos. Por detrás de estas múltiples experiencias subjetivas, se abre un debate sustantivo acerca de las estrategias y objetivos del propio Foro.

El Foro Social Mundial también es el espacio donde se ensayan nuevas formas y recrean nuevas orientaciones para las luchas de transformación. Son búsquedas ambivalentes alimentada por una "... muy flexible coalición de movimientos trasnacionales, nacionales y locales, con múltiples prioridades unidas en su oposición al orden neoliberal. $Y$ estos movimientos en su mayoría, no están buscando el poder del Estado, y si lo están buscando, lo hacen partiendo de que ésta es sólo una táctica entre otras, pero no la más importante". Pero estas mismas fortalezas son sus debilidades. "... La falta de centralización puede hacer difícil coordinar tácticas para las batallas más duras que quedan por delante. $Y$ tendremos que ver también qué tan grande es la tolerancia hacia todos los intereses que se representan, la tolerancia hacia las prioridades de unos y otros". ${ }^{19}$

Construir la tolerancia y el respeto de los diferentes intereses presentes en el FSM es una de las principales estrategias para avanzar en la formulación de alternativas y es, tal vez, lo verdaderamente nuevo que propone un espacio como el FSM. Ninguna centralización organizativa y ninguna agenda de movilizaciones podrán acortar los caminos que se deben transitar para poner en diálogo las diversas prioridades de los movimientos. Las fortalezas y debilidades que se expresan en esta iniciativa y las diferencias explícitas e implícitas que expresan las diferentes corrientes de pensamiento son parte del debate de fondo que el Foro como espacio de confluencia propone.

Desde nuestro punto de vista, el problema central de la articulación de los movimientos sociales no es organizativo sino político y conceptual. Y el desafío, como plantea Boaventura de Souza Santos, "está en la capacidad de formular problemas nuevos para los cuales no existe solución, o no existe aún solución. ${ }^{20}$ Las pistas para esas soluciones se van encontrando y construyendo en el mismo proceso.

\section{Pensar "otro mundo posible"}

Un desafío que enriquece y al mismo tiempo tensiona los espacios de articulación es, en primer lugar, el reconocimiento de cada uno/a como actor/a legítimo/a de esta búsqueda. Sería ilusorio pensar que este reconocimiento es un acto inmediato y "natural" de nuestras "aspiraciones humanistas". El reconocimiento del otro/otra como actor/a de la construcción de un espacio democrático no está fuera de relaciones jerárquicas de poder construidas socialmente, ni de la tensión inherente a la definición del "nosotros - otros".

La política, dice Chantal Mouffe, "tiene que ver con la acción pública y la formación de identidades colectivas. Su objetivo es la creación de un "nosotros" en un contexto de diversidad y conflicto. Pero para construir un nosotros hay que poder distinguirlo de un "ellos". Por eso la cuestión crucial de una política democrática no es cómo llegar a un consenso sin exclusiones o cómo crear un "nosotros" que no tuviera un "ellos" como

\footnotetext{
${ }^{19}$ Immanuel WALLERSTEIN, 2002.

${ }^{20}$ DE SOUSA SANTOS, 2001 , p. 36
} 
correlato, sino cómo establecer esta discriminación nosotros/ellos de una manera que sea compatible con la democracia pluralista". ${ }^{21}$

Esta es la propuesta metodologica del Foro. Por ello, "salvaguardar el derecho a la palabra" y el de "la libertad de los individuos y de los grupos para establecer el sentido de lo que son y de lo que quieren ser" 22 es el aporte sustancial de este esfuerzo por constituir un escenario de actores/as que disputan el significado, las prioridades y los fines de la vida en común.

En segundo lugar, un espacio de confluencia que habilite la tolerancia y el desarrollo de nuevas culturas políticas depende también de la oportunidad para colocar en debate los esquemas de interpretación y significados que los diferentes actores/as otorgan a sus utopías y propuestas.

Es indudablemente un proceso, cuyos tropiezos y dificultades se van encontrando y verbalizando - en el mismo espacio del Foro. En una reunión de evaluación de la metodología utilizada en esta versión del $2003,{ }^{23}$ se analizó el conjunto de las actividades del Foro, tanto las programadas como las propuestas por los movimientos, redes y organizaciones.

Las tareas que cumple el FSM son de movilización, articulación y reflexión y la diversidad, su expresión, incorporación, reconocimiento es uno de los retos del FSM y uno de los ejes de su propuesta político metodológica, que se expresa no sólo en el reconocimiento de voces, rostros, movimientos, estrategias plurales, sino también en potenciar el esfuerzo de reconocimiento de los-las otras y superar la visión jerarquizada de las luchas y movimientos. Escudriñada en sus debilidades y dificultades de articulación, la idea subyacente al enfoque de la diversidad es la importancia de un reconocimiento vital, que sobrepase la tolerancia, por una verdadera "traducción" de concepciones y saberes entre los múltiples movimientos. Y la transformación de la diversidad en divergencia, a ser reconocida y trabajada.

Esta recuperación de las complejidades de la diversidad se alimentó de la realidad de las dinámicas del Foro, donde se aprecia jerarquización de las redes, con poder diferenciado, y jerarquización de temas; donde unos son considerados, explicita o implícitamente, como más importantes que otros. De allí que, celebrando el enorme aporte y trascendencia del Foro Social Mundial, es importante reconocer que también es un espacio de poder, que tiende a reproducir las mismas estructuras de poder de la sociedad. Expresa también lo nuevo y lo viejo en concepciones, prácticas, búsquedas y tensiones. De allí que sólo su reconocimiento explícito puede dar lugar a estrategias explícitas para evitar que impacten como fuerzas ciegas al Foro. Francisco Whitaker lo expresa como un riesgo: "En verdad corremos el riesgo aún de volver atrás. Todo dependerá de la solución que se le dé a algunas de las tensiones actuales del proceso del Foro. Lo nuevo exige cambios en los principales actores de la escena política, partidos y seres humanos, pero "lo viejo" tiene aún fuerza hegemónica en las cabezas y en las prácticas". ${ }^{24}$

Los paneles y las actividades libres fueron los ejes que guiaron la reflexión, en el entendido de que son el centro de la concepción político metodológica del Foro y fue desde su significación y la importancia de su conexión que se analizaron las otras actividades programadas (conferencias, mesas de controversia, testimonios).

\footnotetext{
${ }^{21}$ Chantal MOUFFE, 1996, p. 7.

${ }^{22}$ Alberto MELUCCl, 2001 , p. 57.

${ }^{23}$ Seminario de Sistematización del FSM 2003, Rio de Janeiro. Documento en proceso de edición.

24 , 2003, p. 20.
} 
Si bien los paneles fueron evaluados positivamente en su sentido (el espacio de reflexión desde las apuestas que trae la diversidad), la percepción fue muy negativa en relación com la logística, visibilidad, claridad de sus dinámicas, formas y contenido de las discusiones; así como la forma de funcionamiento de los paneles mismos, desde la definición de los ejes, la metodología, la presencia o ausencia de redes y movimientos, los equilibrios geográficos, regionales, étnicos y sexuales.

Las formas del Foro muestran un formato más bien tradicional de los paneles, con predominio del habla, con falta de alternativas de comunicación. No se procura incorporar lenguajes más universales, que valoricen sensibilidades y emociones, ni se ensayan formatos de mayor horizontalidad y participación.

Las actividades libres (1.715 inscritas en total, de las cuales funcionaron 1.600 aproximadamente) recuperan la diversidad de las miradas y avances de los movimientos y redes. Sin embargo, es imposible no percibir la fragmentación y atomización de estas iniciativas que muchas veces ni siquiera se proponen colocarse en diálogo con otras.

Un análisis realizado por el Comité Organizador (CO) de una muestra de alrededor de 370 talleres u oficinas propuestas, permite rastrear sobre "qué esta conversando la sociedad". Sin embargo, estas actividades tenían la tendencia a ser ensimismadas, sin diálogos transversales, sin incorporación de la diversidad. Y con el agravante de que muchas actividades libres se convirtieron en Foros en sí mismos, encapsulados y sin articulación con las demás actividades libres o con el Foro como tal, negando de esta forma el proprio método propuesto por el Foro, de conexión y traducción entre los movimientos y redes.

La relación entre lo viejo y lo nuevo, entre tradicionalidad y ruptura, es parte constitutiva del Foro, de allí la importancia de ampliar la voz y favorecer la ocupación de espacios para movimientos sociales excluidos (incorporación de los márgenes) o considerados menos importantes, favoreciendo la emergencia de nuevas cuestiones y nuevos protagonistas.

Las propuestas emancipatorias impulsadas por los diferentes actores sociales se desarrollan al interior de relaciones de poder y sus articulaciones no se dan automáticamente. Es posible anunciarlas como un horizonte político democrático pero construirla en la práctica, supone movilizar conceptualmente las jerarquías de interpretación de los problemas a resolver, economía, política, poder mundial, versus subjetividad, diversidad, discriminación, derechos. Al abordar los diferentes problemas parece difícil articular estas perspectivas sin establecer una jerarquía entre los temas. Es por ello que, aún muchos intelectuales y políticos varones prescinden de la teoría de género para integrarla a sus perspectivas de análisis, para las feministas "... no se trata sólo de incorporar a las mujeres como un 'tema' más a investigar, sino de enriquecer los marcos teóricos y conceptuales para lograr un conocimiento más amplio y adecuado sobre el funcionamiento de la economía". ${ }^{25}$

En el FSM se ha avanzado aún escasamente en la premisa de pensar los problemas globales de la humanidad desde una nueva perspectiva emancipatoria que integre y articule lo público y lo privado, las subjetividades y poderes, clase, raza, género, opción sexual, para formular nuevas identidades políticas democratizadoras. Intervenir en este debate es un desafío político para las diferentes corrientes feministas, desde el punto de vista teórico pero también desde la práctica política cotidiana.

Por último, un aspecto central planteado por la existencia misma del FSM es la ampliación del concepto de la políitica, lo políitico y el poder. Al constituirse como un espacio

\footnotetext{
${ }^{25}$ Rosalba TODARO y Regina RODRÍGUEZ, 2001, p. 9.
} 
de encuentro y acción de los movimientos sociales para pensar los problemas y desafíos de la organización actual del mundo, se asume un protagonismo político que amplía el escenario de quienes están convocados/as para tomar la palabra en este debate y proponer cuáles son los asuntos que motivan la búsqueda de felicidad. ¿Qué nuevos puentes entre la sociedad y sus organizaciones, las demandas y problemas, la diversidad y el reconocimiento, plantea este espacio a los sectores políticos progresistas? ¿Cómo abrir y procesar debates entre los movimientos y los partidos sin cooptaciones o exclusiones? ¿Se podrá acortar la brecha entre las ciudadanías diversas y plurales y los espacios de representación política? ¿Qué procesos de democratización de los partidos son necesarios?.

Para las diferentes corrientes feministas estos desafíos adquieren a su vez una dimensión específica, tanto en lo organizativo como en lo políico.

Sin duda el movimiento feminista está compuesto de corrientes diversas, tanto en espacios organizativos como en intereses temáticos y políticos. Parece necesario un mayor grado de articulación entre las diferentes corrientes para no seguir atrapadas, al decir de Marta Lamas, "en rivalidades absurdas, pues la lógica identitaria confronta a compañeras con múltiples coincidencias políticas solo porque pertenecen a redes o instancias distintas. Esos tropiezos, consecuencia de la 'política de la identidad', que favorecen que en los grupos se encaucen inquietudes políticas y vitales sin la necesaria separación entre hacer y ser (Bondi), producen dislocaciones discursivas, falsas oposiciones y confrontaciones personalizadas. ${ }^{26}$

Las alianzas entre las diferentes corrientes y agendas feministas podrían articularse en torno a cómo transformar el debate global sobre las alternativas en un debate de "equivalencias de demandas" y las múltiples estrategias a desplegar para enfrentar tres desafíos básicos, desde mi punto de vista. ¿Cómo eludir la encrucijada en que las "urgencias de las crisis", particularmente la financiera, parece colocar en un lugar secundario las consideraciones de género y su estrecha relación con la economía? ¿Cómo hacer de la cultura de derechos un campo de praxis política? ¿Cómo introducir en los debates actuales la dimensión corporal y sexual de la diferencia?

Las agendas feministas convocan al desarrollo de nuevos paradigmas, combinando lo local, lo nacional y lo global, la interconexión de múltiples agendas y la oportunidad de colocar en debate una dimensión más profunda de la justicia, que integre la justicia económica, social, cultural y simbólica. Pero que también coloque en debate las formas de hacer política de los propios movimientos sociales presentes en el Foro.

Estas interacciones múltiples entre los movimientos sociales podrían implicar algo más que alianzas puntuales o acciones de denuncia, con efectos significativos en las concepciones de autonomía de los movimientos. Parecería que en este posicionamiento en construcción comienza a asentarse la percepción de que los asuntos de las mujeres deben ser colocados como asuntos políticos democráticos de primer orden, que atañen a mujeres y hombres, y que los asuntos de las democracias a nivel cultural, social, económico y político deben ser asuntos de competencia feminista y parte de sus agendas en lo nacional y lo global.

La esperanza abierta por estas búsquedas está de alguna forma respaldada en la presencia activa de los y las actoras sociales que, sin que nadie pueda autorizar o censurarla/ os, deciden participar desde sus sueños, identidades y propuestas.

\footnotetext{
${ }^{26}$ Marta , 2000, p. 91.
} 


\section{Referencias bibliográfícas}

ALVAREZ, Sonia E.. "Latin American Feminisms Go Global: Trend of the 1990's and Challenges for the New Millennium." In: ALVAREZ, Sonia E., DAGNINO, Evelina, and ESCOBAR, Arturo (eds.). Cultures of Politics, Politics of Cultures: Re-Visioning Latin American Social Movements. USA: WestviewPress, 1998. p. 293-324.

BRAIDOTI, Rosi. Sujetos nómades. $1^{a}$ Edición. Buenos Aires: Paidos, 2000.

BUTLER, Judith; LACLAU, Ernesto; ZIZEK, Slavoj. Contingencia, hegemonía, universalidad: diálogos contemporáneos en la izquierda. México: Fondo de Cultura Económica, 2003.

CASTELLS, Manuel. "Globalización, sociedad y política en la era de la información". Analisis Político, n. 37, p. 3-16, mayo-agostol999.

CELIBERTI, Lilian. "Retos para una nueva cultura política". Lola, n. 15, mayo-octubre 2001.

"El movimiento feminista y los nuevos espacios regionales y globales". In: JELIN, Elizabeth (Compiladora). Más allá de la nación: las escalas múltiples de los movimientos sociales. Libros del Zorzal. Buenos Aires, 2003.

CORREA, Sonia. "Globalización y fundamentalismo: un paisaje de género". Suplemento $D A W N$. Documento de la web: http://www.dawn.org.fj/publications/docs/WSFsupport.doc, 20 diciembre 2003.

DAWN INFORMS. "World Social Forum 2002". DAWN Informs. Development Alternatives with Women for a New Era, Fiji Islands, March 2002. Documento de la web: <http:// www.dawn.org.fj/global/worldsocialforum/socialforum.html)>.

DE SOUZA SANTOS, Boaventura. "A crítica da razão indolente: contra o desperdício da experiência. 3 ed. São Paulo: Cortez, 2001.

DOCUMENTO de la Articulación Feminista MARCOSUR sobre la campaña contra los fundamentalismos. Porto Alegre, Brasil, 2002.

FRASER, Nancy. Iustitia Interrupta: reflexiones críticas desde la posición "postsocialista". Santafé de Bogotá: Siglo del Hombre Editores, Universidad de los Andes, 1997.

GRZYBOWSKY, Cándido. "Es posible un mundo más femenino?". In: Foro Social Mundial, enero- febrero. Documento de la web 2002: <www.forumsocialmundial.org.br >.

JELIN, Elizabeth. Diálogos, encuentros y desencuentros: los movimientos sociales y el MERCOSUR. Documento de la web 2001.

JELIN Elizabeth (Compiladora). Más allá de la nación: las escalas múltiples de los movimientos sociales. Buenos Aires: Libros del Zorzal, 2003.

LAMAS, Marta. "La radicalización democrática feminista" In: ARDITI, Benjamín (Editor). EI reverso de la diferencia: identidad y política. Venezuela: Nueva Sociedad, 2000.

LEÓN, Irene (Compiladora). "Retos feministas en un mundo globalizado". Foro Social Mundial, Porto Alegre 2002. Ecuador: ALAI Area Mujeres, 2002.

MELUCCI, Alberto. Vivencia y convivencia, teoría social para una era de la información. Madrid: Editorial Trotta, 2001.

MOUFFE, Chantal. "Por una política de la identidad nómade". Debate Feminista, Año7, v. 14, p. 3-13, 1996.

SAGASTI, Francisco R. "El orden global fracturado emergente". In: Sistema Económico Latinoamericano/Agencia de Cooperación Española. Dinámica de las relaciones externas de América Latina y el Caribe. Buenos Aires: Editorial El Corregidor, 1998. p. 21-52. Publicado también en inglés.

TODARO, Rosalba; RODRÍGUEZ, Regina (Editoras). El género en la economía, Chile: CEM-ISIS Ediciones de las mujeres, n. 32, 2001.

VARGAS VALENTE, Virginia. "Ciudadanías Globales y Sociedades". Nueva Sociedad, Caracas, n. 163, p. 125-138, septiembre-octubre 1999. 
WALLERSTEIN, Immanuel. "Balances del Foro Social Mundial". Documento de la web 2002: $<$ www.forumsocialmundial.org.br $>$.

WATERMAN, Peter. After the 2nd World Social Forum in Porto Alegre: What's Left Internationally?. 2002. Unpublished manuscript.

WHITAKER, Francisco. "O que o Fórum Social Mundial traz de novo como modo de atuação política?" Democracia Viva, Rio de Janeiro: Ibase, n. 14, p. 20, enero 2003. En portugués en el original.

\section{Feminists at the World Social Forum: Challenges for a New Political Culture}

Abstract: The article deals with the ways of incursion of the feminist plurality's significant expressions in the World Social Forum. These incursions express the changes in the subjectivities and in strategies of struggle that feminist movements in particular and social movements in general begin to develop, in the frame of a globalised world and in the new millennium. It's an unprecedented process, that is promoting the development of new paradigms for collective action, that combines local and global issues, the interconnection of multiple agendas and the recovery of a more profound dimension of economic, social, cultural and symbolic justice, broadening, in this process, the concept of politics, the political and the power. The article sets discussion around the ways in which social movements converging in the Forum - that drag old dynamics and at the same time recreate new paradigms - work in politics and open the possibility of re-inventing an emancipation map and a social conception, capable of competing with neo-liberal consensus and with the single thought, recuperating diversity and plurality of social individuals and actors.

Key words: alternative globalisation, political culture, utopia, new paradigms, feminist agendas. 